\title{
التوفيق بين العقل والنقل عند الكندي: دراسة تحليلية
}

\author{
أ. عحمد علي الحمد عبد الصادق \\ جامعة مصراتة / كلية التربية
}

https://doi.org/10.36602/faj.2018.n12.05

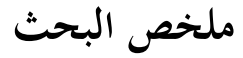

كان للكندي السبق في مسألة التوفيق بين العقل والنقل، فأثبت العلاقة بين العقل والنقل، وسعى إلى التوفيق بين هذين المصدرين بالمنطق للوصول إلى اليقين، وكان ذلك مؤكدًا عند

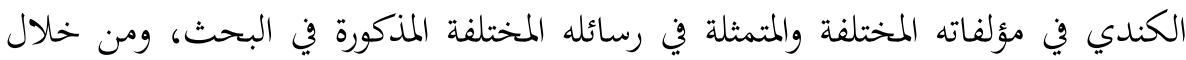
تأكيد الكندي بأن الفلسفة تتم بالعلل والأسباب والعلم بالمعلولات للوصول إلى حقائق ويقينيات، وقد بنى استراتيجيته في الدفاع عن الفلسفة من خلال ما جاء في إشارته بأن الفلسفة تعني الوجود بأنه موجود، وبأن الفلسفة علم الأشياء بحقائقها، وبقدر طاقة الإنسان، كما اهتم بالعقل وحدد مراتبه حيث عرفه بأنه جوهر بسيط يدرك الأشياء

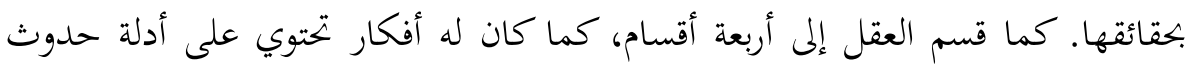
العالم وتناهي الزمان والحركة.

وبشأن مسألة التوفيق بين العقل والنقل ينظر الكندي للدين نظرة عقلية، محاولاً تفسير الدين بأدلة عقلية، وهذا ما دفعه للدفاع عن الدين بأسلوب عقلي محاولا تحاشي أخطاء

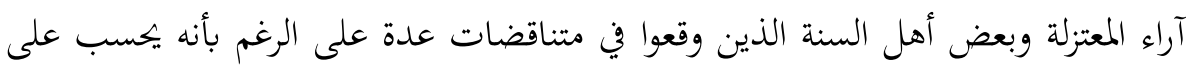
المعتزلة في بداية حياته الفكرية، وكان ينزع عند تفسيره لآيات القرآن الكريم نزعة عقلية، فكان يجتهد في تفسيرها اعتمادًا على عقله، وهذا ما يبعده عن قمة أنه اعتزالي بصورة خالصة؛ لأن الطابع الفلسفي كان يغلب عليه لإيمانه الراسخ بالدين والفلسفة في آنٍ واحد. الكلمات المفتاحية: الكندي- الرأي الفلسني- العقل والنقل - التوفيق- الفلسنة الدينيةالمعتزلة. 


\section{Abestract}

Al-Kindī was among the earliest Muslim intellectuals who meditated about the problematic of Mind and Transference. He proved with brilliant thought the relationship between mind and transference, and sought to reconcile between these two sources with rationality to reach certainty.

This reconciliation attempting was approved in Al-Kindi's various works, represented by the letters mentioned in the research, as well as his believing that philosophy is concerned with the causes, reasons and the knowledge of information to reach facts and certainty. $\mathrm{He}$ divided philosophical science into figures and characters such as theory of rationality and Mathematics. Al-Kindi was interested in the inspiration of mind as a simple essence recognizing things from their facts, set the essence ranks and divided it into four sections.

His ideas contained the evidence of the world's existence, and the emersion of time stage in the matter of reconciling mind and transference. Immersing in an Arab-Islamic environment, $\mathrm{He}$ evaluated religious principles with a mental view, interpreting its concepts with rational evidence. Despite his early affiliation to Mu'tazila, He aspired to interpret the Holy Quran rationally, and tried to interpret Quranic concepts based on reason, which eliminates the suspicion of pure belonging to Mu'tazila, because this philosophical character is due to his firm faith in religion and philosophy at the same time.

Keywords: Al-Kindi; Mind and transference; Religious philosophy; Mu'tazila

1

من أهم الموضوعات التي ركز عليها الفكر الإسلامي منذ القدم التوفيق بين الدين والفلسفة، ولم تنقطع محاولة الفلاسفة المسلمين عن تحقيق ذلك، وكان لفيلسوفنا الكندي السبق في هذا الاتحاه، فاهتم بها اهتمامًا عظيمًا، وخاصة بعد اطلاعه على فلسفة من سبقوه من الفلاسفة سواء المسلمين منهم أو اليونايين كأفلاطون وأرسطو، حيث وجد في 
فلسفاقم آراء تتعارض وتخالف شريعتنا الإسلامية، ولحرص الكندي الشديد على التمسك بالدين الإسلامي والسعي للاستفادة من فلسفة من جاء قبله حول هذه المسألة، فسعى إلى الخوض في موضوع التوفيق بين الدين والفلسفة، وإثبات ما بين النقل والعقل من علاقات همدف التوفيق،حيث اختلفت آراء الباحثين وأكد ذلك الكندي في رسالته المعنونة (رسالة إلى المعتصم بالله) فدافع في رسالته هذه عن الاشتغال بالفلسفة، ويدعو إلى البحث عن الحقائق بغض النظر عن مصدرها، سواء جاءت إلينا من بلاد العرب أم من بلاد الغرب وخاصة اليونان، فيرى الكندي وجوب تقديم الشكر للفلاسفة ولا نقوم بالهجوم عليهم لإفادقم لعقولنا بما قدموه من آراء وأفكار في إطار الفلسفة للوصول إلى الحقائق اليقينية، والتي نستفيد منها في حياتنا ونخن نسعى للحصول على المعارف، ولتجلو لنا كل ما يعترينا من غموض من خلال ما نحصل عليه عن طريق الإلمام والوحي، والتي ينفرد بها الأنبياء والرسل، وما نتوصل إليه كذلك عن طريق الاكتساب والتحصيل والاجتهاد، والتي تبرهن لنا بأن هناك آراء في الفلسفة لا تخالف ما يدعو إليه الدين، وهذا ما دعا الكندي مهاجمة رجال الدين الذين يزعمون بأن الدين لا يسمح بالتفكير العقلي، ويهدف التوفيق بين العقل والنقل والذي يعني جعل الله عبادة وموافقا لما رسمه ويرضاه، والتوفيق هنا يعني الجمع بين الآراء في المذاهب المختلفة، ومحاولة التوفيق بينهما لتكوين مذهبًا واحدًا يمثل الفلسفة الإسلامية.

وسبب اختياري لهذا الموضوع أهميته في مجال البحث، وبعد الاطلاع على ما أتيح

لي من مصادر ومراجع وإن كانت ليست بالقدر الكافي، ولكن لا يمنع ذلك من محاولة تناوله، ولتوضيح الآراء والأفكار والتي تتسم في التوفيق بين الفلاسفة المسلمين وعلى رأسهم الكندي، والفلاسفة الغرب ومن بينهم فلاسفة اليونان الأوائل وعلى رأسهم أفلاطون 
وأرسطو، ولفتح مسارات تتجه من خلالها التوفيق بين ما جاءت به الرسالة السماوية أعني به ديننا الإسلامي- بين ما يؤمن به الفلاسفة. وسأتناول -بعون الله- الجوانب الآتية: الجانب الأول: نبذة مختصرة عن حياة الكندي وأهم مؤلفاته. الجانب الثاني: مفهوم الفلسفة عند الكندي. الجانب الثالث: مكانة العقل عند الكندي ومراتبه. الجانب الرابع: أدلة وجود الله وصفاته. الجانب الخامس: مسألة خلق العالم. الجانب السادس: جوانب التوفيق عند الكندي، وأوصي بأن يكمل غيري النواقص التي لم أتناولها في بحثي لزيادة الفائدة، وما أوتينا من العلم إلا قليلا، ثم أصل إلى لى خاتمة البحث، وأذكر مصادر ومراجع البحث.

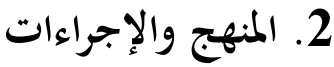

يستخدم البحث الحالي المنهج الوصفي التحليلي، وصولًا إلى استنباط نقاط البحث، وذلك بعد مراجعة عدد من مصادر ومراجع تتعلق بالبحث،و بعد تحديد مشكلة البحث سعيًا للوصول إلى وضع حلول لهذه المشكلة، وسبب اختيار هذه المنهجية هو الوصول إلى معلومات تتعلق بموضوع البحث لتعمّ الفائدة من تناوله، ولتفتح سبلً للغوص في مثل هذه الموضوعات إثراءً للمعرفة المرجو الوصل إليها من خلال تناول مثل هذه الموضوعات الإنسانية.

\section{3. الكندي حياته وآرائه} 3. 1 نبذة عن حياة الكندي:

هو أبو يوسف يعقوب بن إسحاق بن الصباح بن عمران، ابن الأشعت بن قيس، بن الحارث الأصغر ابن معاوية ابن الحارث الأكبر، بن كندة بن يعرب بن قحطان، ولد بالكوفة عام 193ه (محمد، 1994، ص 611)، وهو من قبيلة كندة أي: من أصل 
عربي، ولذلك لقب فيلسوف العرب، وكان أبوه أميرًا على الكوفة، وتحصل الكندي على علومه في مدينة البصرة ثم بغداد، كما اشتغل بترجمة كتب اليونان إلى العربية، وبتهذيب ما كان غيره يقوم بترجمته من تلك الكتب، كما فعل بالكتاب المتحول لأرسطو المسمى (أوتولوجيا أرسطو طاليس)( محمد، 1994، ص 611)، كما اشتغل بقصر الخلافة في بغداد طبيًا، وكان الكندي ملمَّا بعلوم عصره في أيام المهدي، حيث تأدب بعلوم زمانه "ولم يعرف له في بغداد شهرة قبل المأمون، ثم اتصلت شهرته وحظوته في بلاط العباسيين أيام المعتصم" (فروخ، 1981، ص 305). والكندي لم ينل حظًاّ وافرًا من الدنيا ولا صفت حياته من الشوائب، ولذلك اعتزل الحياة العامة "ولا نعرف السبب الحقيقي لطرد الكندي من بلاط العباسيين، ولا السبب الدقيق الذي دعا الخليفة المتوكل بالله العباسي يغضب عليه غضبًا شديدًا، فأمر بضربه ضربًا مبرحًا" (الإمام، 1998م، ص 79-80).

وهذا لا ينقص من المكانة التي وصل إليها إليها: حيث يرى الدكتور: أحمد فؤاد الأهواني واصفًا الكندي: "كان الطليعة المتقدمة في تيار الفكر الفلسفي، أغنى الفكر واستسغناه ورجع عنه بها يستحق بأنه فيلسوف العرب". (الأهواني، د.ت، ص9). ولقد تأثر الكندي بأهل عصره من الفلاسفة الطبيعيي، الذين أخذوا بالمذهب الفيتاغوري الجديد، وكان الكندي كثير الاهتمام بالعلوم الرياضية والطبيعية، كما كان له تجاربه في علم الطبيعيات، وفسّر كثيرًا من أوجه النشاط في الطبيعة تفسيرًا إلهيًّا، كما اشتغل الكندي بالتنجيم لإيمانه به "فكان منجمًّا للرشيد والمأمون" (فروخ، 1981، ص 307)، كما تأثر الكندي بالمعتزلة خاصة في موضوع الإلهيات، فهو لا يميل إلى الجدل ولا الخطابة "ولكنه يميل إلى التعريفات المنطقية والاصطلاحات العامية الدقيقة"( الإمام، 1998م، ص 
79)، فالكندي قد عاش في عصر كان من أثرى عصور حضارتنا العربية المنفتحة، والتي ازدهرت فيه العلوم الإنسانية والتقنية، فتميزت شخصية الكندي الفلسفية بالاستقلالية ولم تكن منغلقة، فتفتحت تلك الشخصية على التراث الفكري الإنساني، كما تأثر الكندي بالتراث الفكري اليوناني "وعلى الأخص الأرسطي، وكان له الفضل في خلق تواصل عميق بين الثقافة العربية الإسلامية آنذاك والتراث الفكري اليوناني (فرحان، 1986، ص 91). ولا شك فإن الكندي فد تأثر بالآراء الدينية الإسلامية؛ لأنه من أعظم فلاسفة الإسلام و تأثر كثيرً بآراء المتكلمين في عصره، ولكنه احتفظ بالمبادئ الأساسية في دينه الإسلامي، فالكندي قد خالف الكثير من آراء فلاسفة اليونان ومن بينهم أرسطو "وخاصة في مفهومه عن العالم والمادة، ويرجع ذلك إلى تأثير المعتزلة على تكوين فكر الكندي الفلسفي" (سليمان، 2005، ص 24). "وإذا تأملنا نزعة الكندي العقلية الفلسفية في فهمه لآيات القرآن الكريم، أو اجتهاده في تفسيرها على مقاييس عقلية، لوجدنا بأن تفكيره يُحسَب على التيار المعتزلي في عصره دون أن يفقد هذا التفكير طابعه الفلسفي وروحه الخاصة" (هناء عبده سليمان، 2005، ص 24). حيث أشاد به يوسف كرم في كتابه تاريخ الفلسفة اليونانية قال فيه: "أحد الذين شاركوا في إقامة العلم، وساعدوا على تنمية قوتنا الفكرية". ( كرم، 1946، ص 208).

ولا تُعرف السنة التي مات فيها الكندي ولا السنة التي ولد فيها، وما يُعرف أن وفاته جاءت بعد أن فقد حظوته في قصر الخلافة، فتوقف القلب الكبير عن النبض، ثم توقف العقل الثاقب عن التوهج، وفاضت الروح إلى بارئها ورجعت النفس إلى خالقها

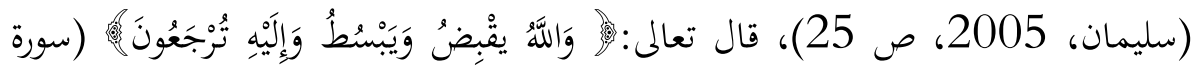


البقرة، الآية: 243)، "وهناك بعض المصادر التي ترى بأن الكندي توفي عام 252هـ،

$$
\text { الموافق 366م" (الإمام، 1998م، ص 79-80). }
$$

والراجح بأن ميلاده كان في أواخر حياة أبيه في الكوفة، والذي توفي زمن الرشيد

المتوفي سنة 193هـ، الموافق 808م، فالكندي "ولد في مطلع القرن الثاني للهجرة حوالي 185، وتوفي حوالي 252 للهجرة، وهو يناهز السابعة والستين من عمره". (آل ياسين، 1983، ص 14). وأهم مؤلفاته:

$$
\text { 3 1. 1. رسالة في حدود الأشياء ورسومها. }
$$

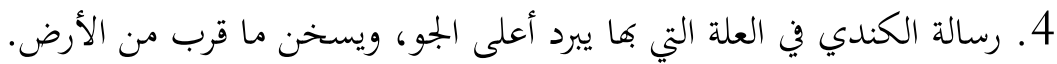
5. رسالة الكندي في علة كون الضباب. تبابل

6. رسالة الكندي في العلة الفاعلة للمد والجزر.

7. رسائل الكندي في العلة التي لهاتكون بعض المواضع لا تكاد تمطر. 8. رسائل الكندي في السبب الذي له نسبت القدماء للأشكال الخمسة. 9. رسائل في القول في النفس وماهية النوم والرؤيا والكلام النفس. 10. رسائل الكندي في أن عناصر الجرم الأقصى كروية الشكل وذلك في علة الكون.

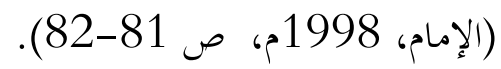

3. 2. مفهوم الفلسفة عند الكندي:

الكندي تأثر كغيره من الفلاسفة العرب والمسلمين في مفهومهم للفلسفة بمفاهيم

من سبقهم من الفلاسفة اليونانيين القدماء، وعلى رأسهم الفيلسوف أرسطو الذي كان له أعظم الأثر في تفكير آراء الفلاسفة المسلمين، فكان هذا التأثير واضحًا في آراء وأفكار 
الكندي في تعريفه للفلسفة في رسالته التي كتبها بتوجيه من الخليفة العباسي (المعتصم بالله)، حيث عرّفها بأنما معرفة الأشياء بما هي عليه في الوجود، وفي رسالته المتعلقة بحدود الأشياء ورسومها، ذكر ستة تعريفات للفلسفة فكان من بينها التعريف الذي يقول بأها: "علم الأشياء الأبدية الكلية من حيث ماهيتها وعللها بقدر طاقة الإنسان"، ويعرفها أيضًا بأها: "علم الأشياء بحقائقها؛ لأن كل شيء له حقيقته، ومعرفة الحق يكون كمال للإنسان"

$$
\text { (الشيباني، 1975، ص 71) (7) }
$$

وعند تدقيق النظر في هذا التعريف نجد الكندي يقدر قيمة الحق وإعلاء شرفه،

وتمثل هذه النظرة للفلسفة خاصية جميلة تتجلى عند فيلسوفنا، وتوضح الحكمة العظيمة في فكره الثاقب لتعريفه للفلسفة كما أوضحت عن الروح العربية الإسلامية مثل عبارة "الحكمة ضالة المؤمن، وخذ الحكمة لا يضرك من أي إناء خرجت" (الشيباني، 1975، ص 7172)، وكما جاء في تعريف الفلسفة القائل "هي تمكن الأشياء بحقائقها بقدر طاقة الإنسان، وفي هذا العلم توجد علوم كعلم الربوبية وعلم الوحدانية وعلم الفضيلة، وجملة في كل علم نافع وأوضح السبيل إليه وبين البعد عن كل ضار والاحتراس فيه" (المهدلي، 1988، ص 81-81)

وكذلك نجد للكندي تعريفًا آخر للفلسفة يقول: بأها صناعة الصناعات وحكمة الحكم"، وهذا يتطابق مع تعريف أرسطو لها حين عرّفها بأن الفلسفة وعاء العلوم وأمها ومغذيها، كما نجد تعريف المعز الكندي للفلسفة يقول فيه: معرفة الإنسان نفسه، ويرى بأن معرفة الإنسان نفسه ليست هاية المطاف أو الغاية، بل يراها وسيلة لمعرفة العالم الأكبر، أي المعرفة بالطبيعة والكون الذي يوجد فيه الإنسان، حيث قال: "لا تتوقف المعرفة عند هذا الحد وإنما ترتقي من معرفة العالم إلى معرفة خالقه" (الشرقاوي، 1980، ص 102). 
و تأثير الفلسفة اليونانية وخاصة أرسطو في تعريف الكندي للفلسفة واضحًا كما تأثر بالإمبراطورية المحدثة والفيتاغورية الجلديدة وخاصة في قوله: "لا يكون الإنسان فيلسوفًا حتى يدرس الرياضيات"، وفي قوله كذلك: "لا تنال الفلسفة إلا بعد الرياضة" (السيد محمد المهدلي، 1988، ص 84). والفلسفة عند الكندي تَتم بالعلل والأسباب، والعلم بالمعلولات، "وما دام الله هو السبب الأول للأسباب كلها، فإن الفيلسوف الكامل هو الذي يسعى إلى معرفة الله وما يتعلق بالإلهية من صفات الكمال" (الشرقاوي، 1980، ص 103). قسّم الكندي الفلسفة إلى قسمين: قسم نظري وآخر عملي، وهذا التقسيم مستمد من تقسيم أرسطو، القسم النظري يرجع إلى غرض الفيلسوف أي إصابة الحق، فهو علم الأشياء بحقائقها، ومنه علم الربوبية وعلم الوحدانية، والقسم العلمي: الذي يكون غرض الفيلسوف منه هو العمل به، فهو علم الفضيلة، والطريق الذي رسمه الكندي أمام الفيلسوف لمعرفة الحقيقة، وهذا يتفق والأفلاطونية، فهو يذكر بأن الروح الفلسفية أساسها الحب والنور وتمجيد قوى العقل والقلب، وعلى الدارس للفلسفة أن يتعلم الحساب والهندسة والفلك والموسيقا، وينتهي بالجدل الذي هو أسلوب معرفة الفلسفة. وقسم الكندي العلم الفلسفي إلى أقسام: رياضيات، منطق، طبيعيات، ميتافيزيقا، أخلاق، سياسة، وأما بشأن العلم الديني فهو العلم الذي تبحث فيه أصول الدين والعقائد والتوحيد، وكذلك الرد على المبتدعة والمخالفين، ولم يُهمِل الكندي الطب والكيمياء، ولكنه لمج يُحِّدد مكانة هذين العلمين في تصنيفه، كما أنه لم يبيّن إلى أي القسمين الرئيسيين ينتسبان، "وأشرف العلوم الفلسفية عند الكندي وأعلاها مرتبة الفلسفة الأولى أعني علم الحق الأول الذي هو علة كل حق" (المهدلي، 1988، ص 82). ولذلك يجب أن يكون 
الفيلسوف هو المرء المحيط بهذا العلم؛ لأن علم العلة عند الكندي أشرف من علم المعلول؛ لأننا إنما نعلم كل واحد من المعلومات علمًا تامَّا، "إذن نحن أحطنا بعلم علته، وهذا العلم يسمى أيضًا بالميتافيزيقا" (المهدلي، 1988، ص 82-83). كما قال الكندي في علوم الفلاسفة: "إن علوم الفلاسفة والعلوم البشرية العادية إنما تأتي ثمرة لتكلف البحث والحيلة والقصر إلى المعرفة، والاعتياد بالرياضيات والمنطق طبقًا للمنهج العلمي والفلسفي" (المهدلي، 1988، ص 83)، فالكندي كان مدافعًا عن الفلسفة دفاعًا مستميًًا، وتحمل في سبيل ذلك ضروبًا من الأذى والاضطهاد، كما تحمل الإهانات من طائفة من الفقهاء المتصفين بضيق الأفق وقلة الفهم، ورد عليهم الكندي ردًا مفحمًا، واتمهم بالمتاجرة بالدين، بل نعت بعضهم بالكفر والتنكر للحقائق الثابتة، ويبدو بأن الكندي كان هدفًا لهذه الطائفة والتي كانت بها صلة بالمعتزلة ولقلة وعيةم بالفلسفة، بل يظنون بأن الفلسفة علم دخيل جاء من بلاد الكفر والوثنية، ويقصدون بلاد اليونان وفارس، وربما جاء الهجوم على الفلسفة من بعض الأطباء النصارى كابني موسى بن شاكر لعدم إيمافم بأن الإنسان العربي المسلم قد يتفوق في علوم الطب والفلسفة "ومن هنا كانت دوافعهم الشخصية والذاتية في المقام الأول" (الإمام، 1998م، ص 84). وقد بنى الكندي استراتيجيته في الدفاع عن الفلسفة من خلال الإشادة بالفلسفة لشرف موضوعها، وكل صناعة إنما تحدد مرتبتها على أساس الموضوع الذي تتخذه هدفًا للدراسة، وموضوع الفلسفة هو الوجود من حيث انه موجود كما ذكر الكندي معرًّا للفلسفة بأها علم الأشياء بحقائقها وبقدر طاقة الإنسان؛ لأن غرض الفيلسوف الحقيقي هو: إصابة الحق في حياته، ولا يتأتى الحق إلا بالوقوف على حقائق الأشياء؛ لأن حقائق 
الأشياء هي أسباجها وعللها؛ لأن علة وجود كل شيء وثباته هو الحق؛ لأن كل حالة آتية له حقيقة. ومن علوم الفلسفة عند الكندي الفلسفة الأولى وعنده في الذروة من العلوم قاطبة؛ لأن الفلسفة هي أشرف العلوم؛ فموضوعها هو الذات الإلهية وجودها وصفاقا وأفعالها، وإلى جانب ذلك فهي تحتوي على مجامع الفضائل ومكارم الأخلاق، ويبيّن الكندي بأن دراسة الفلسفة لازمة وضرورية لأنصار الفلسفة وخصومها على حد سواء، ويؤكد أرسطو ذلك بقوله: "من أراد نقض الفلسفة فعليه بعلم الفلسفة؛ ذلك لأن الإنسان يكتاج إلى علم الأدلة والبراهين لكي ينقض الفلسفة، وهذا العلم من صميم الفلسفة!! وذلك لأن إعطاء العلة والبرهان من علاقة علم الأشياء لحقائقها"( الإمام، 1998م، ص .$(86$

\section{3 العقل ومراتبه عند الكندي:}

عرّف الكندي العقل بأنه "جوهر بسيط يدرك الأشياء بحقائقها، والعقل الإنساني له طاقة وقدرة محددة ينبغي أن يقف عندها"، ونجد الكندي لا يثق في العقل ثقة مطلقة كما فعل أرسطو، ولكنه أدرك أن العقول متفاوتة، وهذا يعتبر دليل على أن لكل عقل قدرته وطاقته.

والعقل عند الكندي ينقسم إلى أربعة أقسام: أولها: العقل الذي هو بالفعل دائمًا،

وهو علة جميع العقول والمعقولات، ويصفه بالعقل الأول، وثانيها: العقل الذي هو في نفس الإنسان بالقوة، وثالثها: العقل كعادة وهو الذي في النفس بالفعل، ونستطيع استعماله متى شئت كقوة الكتابة لدى الكاتب، ورابعها: العقل هو فعل به تطهر النفس ما هو فيها بالفعل، والعقل الأخير عند الكندي فعل الإنسان ذاته، "أما إخراج القوة إلى العادة أو 
خروج العقل من القوة إلى الفعل فيرى الكندي بأنه من فعل العلة الأولى، أعني العقل الذي هو بالفعل دائمًا" (دي بور، 1981، ص 185-186). ويرى الكندي أن العقل الذي يخرج من القوة إلى الفعل هو هبة من الله، ولذلك فإن العقل الثالث يسمى بالعقل المستفاد، وهذا الرأي الذي قال به الفلاسفة القدماء، و بأن كل ما نعرفه عن الموجودات من مصدر خارج عنها تفيد في ثنايا الفلسفة العربية في صورة العقل المستفاد، ثم سرى إلى الفلسفة المسيحية. وهنا نجد الكندي قد تأثر بفلسفة الإسكندر الأفروديسي في نظرية العقل عنده، كما نجد الكندي قد تحدث عن النفس ووصفها بأها جوهر بسيط محبوسة في الجسد، وعند تخلصها من جسدها فهي كانت أقرب إلى النفس الإلهية الخالصة، وهذا ما يجعل النفس الإنسانية في نظره محدودة الآفاق والمعرفة، ويكون سمو النفس في محاربة الشهوات، وتكون زاهدة في عالم المحسوسات والتكامل والاستغراق في عالم المعقولات، وهذه النظرة الصوفية المثالية عند الكندي، "فوفق بين آراء أفلاطون من جهة والقرآن الكريم من جهة أخرى" (محمد، 1971، ص 122). كما نجد الكندي في تناوله للعقل قد تأثر بآراء أرسطو المختلطة بالأفلاطونية ولم تشتمل على إبداع جديد، وخاصة في رأيه بأن العقل يدرك الكليات المعنوبة الثابتة بين الحواس تدرك الجزئيات المادية المتغيرة، وبين الحواس والعقل قوة التخيل المتوسطة، وملخص رأي الكندي في العقل العقل الفعال الذي هو الله علة كل العقول الأخرى، ويرى بأن العقل الخاص بالنفس الإنسانية والموجود فيها بالقوة مثل عقل الطفل. وأما العقل الثالث: فهو عنده العقل الموجود في النفس بالفعل ويصفه بالعقل الناجح، والعقل الرابع والأخير: ذلك العقل الذي تفصح به النفس عن مكوها الداخلي. 


\section{4 أدلة وجود الله وصفاته:}

تُعدُّ دراسة الكندي للمشكلات الألوهية استمرارًا لموقفه الاعتزالي، ونجد لهذا الموقف في رسالتيه بعنوان: "في الفلسفة الأولى"، و"في وحدانية الله وتناهي جرم العالم"، فهو يرى بأن الله من حيث طبيعته هو الأنية الحقة، فالله هو الوجود التام الذي لم يسبق وجود ولا ينتهي له وجود، ولا يكون وجود إلا به، ومن حيث الصفات واحد تام، فالوحدة من أخص صفات الله، إذ هو واحد بالعدد واحد بالذات، وواحد في فعله بحيث لا يمكن أن

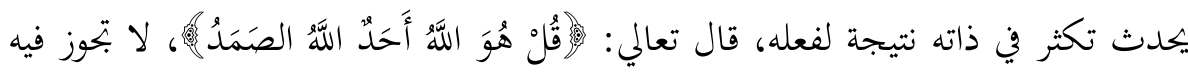
الكثرة لأنه ليست هيولى أو صورة أو كمية أو كيفية أو إضافة، وليس له جنس أو فصل أو شخص أو خاصة أو عرض عام، فهو ليس متحرًا ومن ثم فهو وحدة مضضة. وكذلك هو أزلي أي: أنه ليس هناك ما هو أقدم منه، وكذلك لا يستمد وجوده من غيره، وكذلك ما لا يستمد من علة له ولا موضوع له ولا بجهول ولا فاعل ولا سبب، وهو كذلك "لا يخضع للفساد ولا يتحرك، ومن ثم فإنه ليس له زمان؛ لأن الزمان عدد الحركة"

$$
\text { (أبو ريان، 1992، ص224-225). }
$$

ولهذا الموجود الأزلي فعل خاص به، أي الإبداع "فالله هو العلة الأولى وهو الفاعل

$$
\text { الأول المتمم لكل شيء ومؤسس الكل عن ليس" (أبو ريان، 1992، ص 227). }
$$

وبراهين الكندي على أدلة وجود الله وعلى وجود الحركة والكثرة والنظام كما فعل أرسطو وغيره من الفلاسفة اليونايين، فكان برهانه الأول يستند إلى صفة الحدوث: فالشيء المحسوس في نظره لا يمكن أن يكون علة لذاته، إذًا فالعالم حادث وله بداية في الزمان، ومن كَََّّ فلا بد له من محدث، ومحدثه هو الله، والدليل قائم على ارتباط فكرة العلة بفكرة الحدوث، وله دليل آخر على وجود الله القائم على كثرة الموجودات، فيرى أنه لا يمكن أن 
تكون في الأشياء كثرة بلا وجوده، ولا وجوده بلا كثرة في كل محسوس، وملا كانت المحسوسات كلها مشتركة في الكثرة والوحدة كان ذلك عن علة لا عن صدفة، وهذه العلة هي أمر آخر عن الأشياء مشتركة في الوحدة والكثرة؛ لأنه لو كان غير ذلك لاستمر بنا التناسل إلى ما لا هاية، فلا يبقى إلا أن يكون لاشتراكها علة أخرى غير ذاتما أرفع وأشرف منها وأقدم، إذ العلة قبل المعلول بالذات.

أما البرهان الثالث على وجود الله فهو قائم على التدبير، وهو برهان الغائية في الكون الذي أشار إليه أرسطو ومضمونه أن العالم المرئي الذي لا يمكن أن يكون تدبيره إلا بعالم لا يرى، "والعالم الذي لا يرى لا يمكن أن يكون إلا بما يوجد في هذا العالم من التدبير والآثار الدالة عليه" (أبو ريان، 1992، ص 227).

ويتناول الكندي صفات الله حيث يُعدُّ البحث من صفاته تعالى من أهم ما اهتم به

الكندي؛ لأن غرض كل علم إصابة الحق والعمل بوحيه، وملا كان الله هو على كل حق، وهو الشيء الأيقن حيث ذكر في صفاته:

أ- وحدة الله وأزليته أول صفة من صفاته تعالى، ويعرف الأزلية فهو لا يحتاج في قوامه لغيره "والذي لا يحتاج في قوامه لغيره فلا علة له، ومالا علة له فدائم أبدًا أنه يكون ضروري الوجود أو موجود دائمًا ولم يكن معدومًا" (سليمان، 2005، ص 68-69). ب- صفة الوجود بالذات: يرى الكندي في هذه الصفة بأن الله موجود بذاته، ولم يأخذ وجوده أو يعطى الوجود له أو يوجد آخر وهو ما يعرف بواجب الوجود لذاته أو بذاته. ج- صفة وحدانية الله: يقرر في هذه الصفة بأن الله واحد وأنه الواحد بالحقيقة وما سواه فوحدته عرضية. 
ويرى بشأن الكلي والجزئي فالفلسفة تطلب الكلي، ثم يتم تقسيم ذاتي وعرضي، بحيث يصل إلى أن كل ملفوظ له معنى فإما أن يكون حسًّا أو صورة أو شخصًا أو فصلًا. 3. 5 أدلة الكندي على حدوث العالم:

توصل الكندي إلى إثبات حدوث العالم عن طريق المقدمات والأصول التي وضعها أساسًا لآرائه واستنتاجاته التي أدت به إلى الاتفاق مع عقيدته الإسلامية، وبالرجوع إلى رسائل الكندي الفلسفية نجده يتناول مسألة حدوث العالم وقدمه، وخاصة عند تحدثه عن حدوث الأشياء وتناوله للوحدانية وتناهي جرم العالم، حيث نجده في هذه الآراء متأثرًا بآراء المعتزلة وعلى سبيل الخصوص التصريح بفكرة تناهي الزمان وتناهي الأشخاص؛ لأنه اعتمد عليها اعتمادًا كبيرًا؛ لأنه كان يذكرها في مواضيع عدة من رسائله بعد أن أقامها على أساس فلسفي، وهنا يهمنا بيان دليل الكندي المستند على إثبات تناهي جرم العالم:

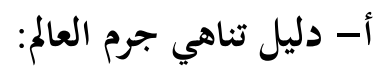
كانت نقطة البداية التي انطلق منها لتأكيد حدوث العالم هي محاولته البرهنة على تناهي جرم العالم، والذي كان يقصد به إقامة الزمان والحركة، فهي دليل على هذا التناهي واللاتناهي عنده، فيعني ذلك لا أول لوجوده، وهذه الفكرة تتطابق بأفكار المعتزلة في تأكيدهم بفكرة التناهي، أي تناهي الأشياء؛ وبالتالي "إثبات البداية في كل شيء سواء كان جسمًا أم زمانًا أو حركة، وذلك لإمكان إثبات حدوث العالم" (سليمان، 2005، ص

ومن مقدمات الكندي على إثبات تناهي جرم العالم ذكره لبعض البديهيات الرياضية، وين قوله: "فإن كان متناه حادث"، وأيضًا قوله: "إن كل ما في جرم أو محمول على جرم متناه"، وهذا عنده يشمل الزمان وحركة الجرم، ومن خلال هذه المقدمات نجده 
يقول بأن المقدمات الرياضية كثيرة، وهي في الحقيقة قضايا بديهية وبينة بنفسها وواضحة ومعقولة، وهذه المقدمات قد توصل بها الكندي إلى فرضيات تثبت آراءه في ذلك، حيث يكون اللامتناهي أكبر من اللامتناهي "ويكون الكل مثل الجزء، وهذا تناقض، إذن فلا بد أن يكون الجسم الموجود بالفعل متناهي، وأن يكون جرم العالم متناهيًا تبعًا لذلك" (سليمان، 2005، ص 53). ب- دليل تناهي الزمان والحركة: يُعدُّ البحث في الزمان من البحوث التي تحتل أهمية كبيرة في تاريخ الفكر الفلسفي خاصة الإسلامي، إذ البحث في هذا الموضوع قد ارتبط ارتباطًا مباشرًا بمسألة الوجود بوجه عام؛ لأن الزمان عامل جوهري مقوم للوجود، وفي الواقع لا يدلل الكندي إلا على بداية

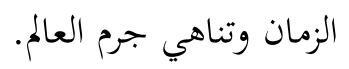

لم يرتب على بداية الزمان تناهي جرم العالم وحركته، ويرتب على تناهي جرم العالم بداية الزمان وحركته، ولكن عندما تثبت بداية للزمان تثبت معه بداية الحركة. وكذلك إذا أثنتنا تناهي الجرم ويكون قد أثتننا بداية الحركة، وبالعكس لأنه لا حركة إلا في جرم، ولا جرم إلا وهو متحرك. والكندي يؤكد بأنه لا جرم بلا زمان، لأن الزمان إنما هو عدد الحركة أي مدة تعدها الحركة، فإن كانت حركة كان زمانًا، وإن لم تكن حركة لم يكن زمانًا، والحركة إنما هي حركة الجرم، فإن كان جرم كانت حركة، وإن لم يكن جرم لم تكن حركة، "والحركة هي تبدل الأحوال وكل تبديل فهو عادة عدد مدة الجرم، فكل تبدل فهو لدى الزمان، فإن كانت حركة كان جرم اضطرادًا" (سليمان، 2005، ص صوس 57). 
وخلاصة القول: إن الكندي يؤكد في فلسفته أن العالم حادث ومخلوق، وهو في هذا يخالف أرسطو الذي قرر أن العالم قديم غير مخلوق، وخلق الله للعالم عند الكندي تأكيد لما جاء في القرآن الكريم، وهذا الخلق عند الكندي تم في غير زمان وبفضل قدرة مبدعة كانت العلة الأولى للخلق وهو الله، وبرغم مخالفة الكندي لأرسطو في هذا فإنه تأثر منه بفكرة أن الجسم والزمان والحركة كلها مرتبطة في هذا الوجود دون أن يسبق أحدها الآخر، ثم يزيد على ذلك بقوله: "إنها حادثة لها بداية وكذلك هاية، مخالف بذلك أرسطو واتفق مع روح الإسلام وآيات القرآن الكريم لكن بطريقة فلسفية" (عمد، 1971، ص 121). حيث ذكره أحد معلميه الإمام علي موسى الرضا بأنه كان محبَّا للفلسفة حيث قال: "أخذ عنه الفلسفة والعلم وحرية الفكر". (سيد أمير علي، 1938م، ص 37). مع رفض الكندي معظم آراء أرسطو في قدم العالم، إلا أنه تأثر بآراء أفلاطون

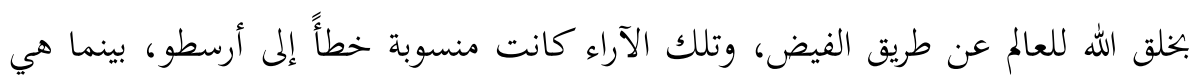
امتداد لآراء أفلاطون، لدى أفلوطين، فنظرية الفيض الأفلاطونية لا تتفق تمامًا مع ديننا الإسلامي؛ لأن الله خلق العالم دون أن يفيض به، وبالتالي أخذ الكندي بفكرة الخلق، ورفض فيض العالم من الذات الإلهية، لأن الله خلق العالم دفعة واحدة وبقدرة إلهية مبدعة.

\section{6 جوانب التوفيق بين العقل والنقل عند الكندي:}

الكندي رجل دين لأنه عربي مسلم، وعاش في بيئة عربية إسلامية متدينة، ويي ذات

الوقت كان فيلسوفًا مبدعًا في عصره ونظريته العقلية الثقافية متفتحة على الفلسفة اليونانية في عصره، وجعله ذلك ينظر للدين نظرة عقلية محاولًا تفسيره، وساعيًا للدفاع عنه بأسلوب عقلي جديد، كما نجده يتحاشى أخطاء بعض آراء المعتزلة وبعض أهل السنة الذين وقعوا في متناقضات عديدة من وجهة نظر الكندي، مع العلم أنه كان معتزليّا بجددًا في بداية 
حياته الفكرية فكان ينزع في فهم آيات القرآن منزعًا عقليَّا، فكان يجتهد في تفسيرها اعتمادًا على عقله والتي لا يكون فيها خلاف، فكان يحاول تأكيد الجوهر العقلي في الدين الإسلامي، وهنا نجده لم يكن معتزليّا خالصًا؛ لأن الطابع الفلسفي يغلب عليه، وهذا الذي أدى به لأن يتحول إلى فيلسوف مسلم،و هذا يتضح من خلال رسائل الكندي ومؤلفاته العديدة والتي تبرهن تفسيراته لآيات القرآن تفسيرًا عقليَّا. والذي دفعه إلى التوفيق بين الدين والفلسفة أن القرآن الكريم يدعو إلى النظر والبحث في أغوار الكون، وهذا ما دفع فلاسفة العرب والمسلمين إلى القول بأن الفلسفة لا تتعارض مع الدين. فكان يُنظر إلى الفلسفة في زمنه نظرة شك وإدانة، وخاصة في عصر المتوكل، حيث قوي نفوذ أهل السنة كما ساند المتوكل الكندي على خلاف المأمون الذي كان مساندًا للمعتزلة، وهنا وجد الكندي لزامًا عليه الدفاع عن النظر العقلي الفلسفي، وبسبب هذا الموقف قد لحقه الأذى بسبب اشتغاله بالفلسفة، ومع حرصه على موقفه المناصر للفلسفة حتم عليه الدفاع عن موقفه الذي لزمه، وهو التقريب بين الفلسفة والدين؛ لأنه كان يرى الفرق الأساسي بين الفلسفة والدين هو الطريق في كل منهما للحصول على العلم ومصدره، كما كان يرى بأن علوم الأنبياء بلا طلب بل بإرادة الله، بينما علوم عامة البشر يجصل بالاكتساب والتدريب والتجربة والبحث، أو كما نجد القرآن الكريم قد حمل بآياته العديد من الأخبار والمعلومات من الخلق والخالق، وحرك للعقل الإنساني للإتيان بتفصيلاتما، وهذا يرمي إلى إيقاظ العقل الإنساني من غفلته لينظر ويتأمل في الحقائق محاولاً

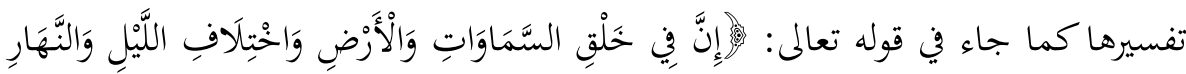

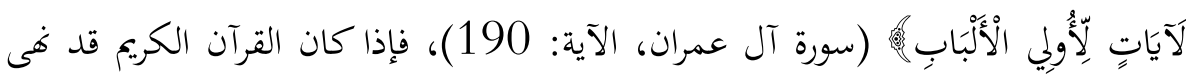
عن الجدل في أمر العقيدة، فهو لم يكن ضد التفكير والتأمل والنظر وهو السبيل إلى معرفة 
الله، وبالتأمل والاستدلال على وجود الله في العالم المخلوق فدعانا الله دعوة صريحة للنظر في

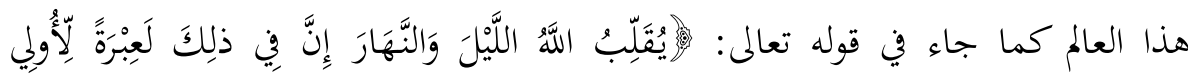

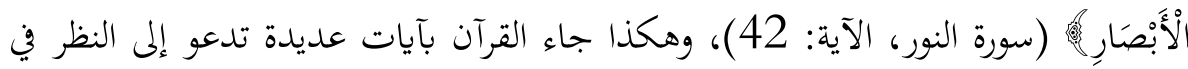
الموجودات، وتدعو إلى التعقل والتفكير؛ لأن الحكمة في القرآن الكريم ما هي إلا الفلسفة. ولكننا نجد فضلًا على الكندي من قِبل الخنليفة المأمون، حيث شجعه ودفعه إلى الاهتمام بالفكر الفلسفي، حيث قال عنه: " بطبيعته العقلانية كان يناصر النهضة الفكرية، إذ كان متفوقًا على معظم رجال عصره" ( آل ياسين، 1983م، ص 3). ومن هنا ابتحه الكندي إلى تلك الآيات التي تحمل الحقائق في هذا العالم وما به من مخلوقات دالة على قدرة الله، فأفاض في تفسيرها وأبان دلالاتا، "كما أكد الكندي بأن استعمال العقل هو مبدأ إسلامي وليس مقصورًا على الفلسفة اليونانية، أو منقولًا عنها أو عن غيرها من الفلسفات" (سليمان، 2005، ص 339). كما حث القرآن الكريم الإنسان إلى أعمال عقلية في السعي لاكتشاف قوانين الحلقة وآيات الإعجاز يبرهن على عظمة الخالق، وهذا ما دل على أن القرآن الكريم كان عاملاً هامًا في إنارة العقل، بعد تطهير عقله من الاعتقادات الباطلة الموروثة والتي سبقت نزول القرآن الكيم، حيث جاءت في تصورات تنزع إلى تفسير العالم وخلقه تفسيرًا أسطوريًّا، أو تصورات ترمي إلى الوثنية والشرك، وإلى غير ذلك من المنازع المادية في تفسير وجود العالم كما جاء في آيات القرآن الكريم في دعوتا الإنسان إلى طرح التقليد الأعمى وتحرير عقله

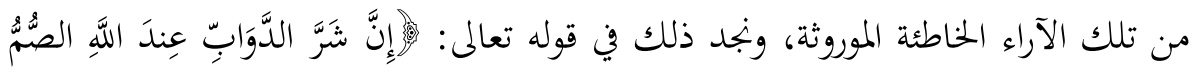
الْبُكْمُ الَّذِينَ لَا يَعْقِلُونَلَهُ (سورة الأنفال، الآية: 22)، وهذا يعني أن الله تعالى جعل الذين لا يستخدمون عقولهم منزلتهم في مرتبة البهائم بل أكثر منها شرًا؛ لأن الله ميز الإنسان عن بقية خلقه بالعقل الذي هو مبدأ كماله وشرفه. 
ومن ذلك كله نجد الكندي يهتم بإبراز أن العقل لا يتناقض مع النقل كما اهتدى به الفلاسفة الإسلاميين الذين جاءوا بعده أمثال: الفارابي، وابن سينا، وابن رشد، وغيرهم، ويرجع الفضل للكندي في توجيه الفلسفة الإسلامية وصهر التوفيق بين الدين والفلسفة، لأهما يطلبان الحق، وإن الدين يعتمد على الوحي، والفلسفة تعتمد في إحقاق الحق إلى المنطق، فالمنطق علم يحفظ عقل الإنسان من الوقوع في الخطأ، فالحق هو القاسم المشترك بين الدين والفلسفة. كما كان للكندي موقف من المتاجرين بالدين والمسيئين له فقال عنهم: "الدين منهم براء، وهم أبعد فئة عن قول الحق وانتهاج سبيل الصدق، والأخذ بمنطق اليقين" (رسائل الكندي الفلسفية، 1950م، ص 8، 14، 14).

\section{4. الاستنتاجات}

الكندي فيلسوف عربي مسلم، كان له الفضل في إبراز أهمية التوفيق بين العقل والنقل، بعد اطلاعه على فلسفات من سبقه من فلاسفة اليونان، والعرب، والمسلمين، أمثال: أفلاطون، وأرسطو، وفلاسفة الفيتاغورية، كما كان له إسهامات في مجال الطب، وخاصة من أيام المأمون والمعتصم، ولكنه لم ينل حظًّ وافرًا من الدنيا؛ لأنه تعرض لكثير من المضايقات، وخاصة زمن المتوكل بالله العباسي، بل ضُرب وأهين زمنَ حكمه، وكان له اهتمامات بعلوم عصره كعلم الطبيعيات والموسيقا. و تأثر الكندي بآراء المعتزلة، وخاصة في موضوع الإلهيات، ولكن كان ميله إلى التعريفات المنطقية، والاصطلاحات العلمية الدقيقة، فالكندي قد عاش في عصر كان الترف متفشيًّا، فكان يميل إلى الاستقلالية، ولم يكن من المنغلقين في آرائه الفلسفية، حيث كان له الفضل في خلق تواصل بين الثقافة العربية الإسلامية والتراث الفكري اليوناني، كما 
كان له تأثر بآراء المتكلمين في عصره، ولكنه كان يُغَلِّب المبادئ الأساسية في دينه الإسلامي، مما جعله يخالف آراء الفلاسفة اليونانيين، وخاصة آراء أرسطو، وخاصة في مفهومه عن المادة والعالم، وهذا راجع إلى تأثير المعتزلة على تكوين فكره الفلسفي، وخاصة في فهمه لآيات القرآن الكريم، واجتهاده في تفسيرها على مقاييس عقلية، فكان يرى معرفة الإنسان نفسه وسيلة لمعرفة العالم كونًا وطبيعة، وهذا يوصله- في نظره- إلى معرفة الله، ولكل ما يتعلق بالألوهية من صفات الكمال. فالفلسفة عند الكندي موضوعها الوجود، وبالتالي فعلة وجود الشيء، وإثباته عنده هو الحق، فكل ما له أنية له حق، فموضوع الفلسفة هو الذات الإلهية، وجودا، وصفات، وأفعالا، كما تحتوي على بجامع الفضائل ومكارم الأخلاق. والفلسفة- عند الكندي- قسمان: نظري، وعملي، وهذا التقسيم نجد الكندي قد تأثر فيه بآراء أرسطو، فغرض الفيلسوف في القسم الأول إصابةٌ الحق، فهو علم الأشياء بحقائقها، فمنه علم الربوبية، وعلم الوحدانية، أما القسم الثاني العملي، فغرض الفيلسوف منه هو العمل به، أي: علم الفضيلة، فالكندي في هذه الآراء قد تأثر بالأفلاطونية، وخاصة في سعيه إلى الوصول إلى الحقيقية؛ لإيمانه بأن سعي الفيلسوف لمعرفة الحقيقة مطالب للقيام به، فالروح الفلسفية أساسها الحب، والنور، وتمجيد قوى العقل والقلب. كما قسم العقول إلى أربعة أقسام: أولها العقل الذي هو بالفعل دائمًا، وثانيها العقل الذي هو في النفس الإنسانية بالقوة، والثالث: العقل كعادة، وهو الذي في النفس بالفعل، ورابعها: العقل الذي هو فعل تظهر النفس ما هو فيها بالفعل في الجسد، وهنا نجد الكندي متأثرًا بآراء الفلاسفة اليونان، وخاصة أفلاطون، وأرسطو. 
كما كان له أدلة لوجود الله وصفاته، فهو متأثر بآراء المعتزلة، فهو يؤكد وجود الحركة والكثرة والنظام، فبرهانه يستند إلى صفة الحدوث والتدبير، أي: برهان الغائية في الكون، كما بيّن بأن وحدة الله والوجود وغيرها من الصفات التي يصف بها الذات الإلهية. كما كان للكندي أدلة لحدوث العالم، منها: دليل التناهي لجرم العالم، وتناهي الزمان والحركة، فنجده في ذلك قد خالف آراء أرسطو؛ لأن أرسطو يرى بأن العالم قديم، ووافق آراء أفلاطون في خلق العالم عن طريق الفيض، ولكن لم تكن الموافقة بصورة تامة مع ديننا الإسلامي؛ لأن الله خلق العالم دون أن يفيض به. بيّن الكندي ما جاء به القرآن الكريم من آيات تدعو الإنسان إلى النظر والتدبر في الموجودات، وتدعو كذلك إلى التعقل والتفكير، فالحكمة في القرآن ما هي إلا الفلسفة، فاستعمال العقول عند الكندي هو مبدأ إسلامي، وليس مقصورًا على الفلسفة اليونانية، أو منقولًا منها، أو عن غيرها من الفلسفات، فيرجع الفضل إلى القرآن الكريم في دعوة الإنسان إلى إعمال العقل سعيًا لاكتشاف القوانين الموصلة للحق، وهنا يبرز لنا بأن القرآن الكريم، والدين الإسلامي عمومًا، كان عاملاً مهمَّا في إثارة العقل بعد تطهيره من المعتقدات الباطلة والموروثة، حيث نجد الكثير من آيات القرآن تدعو الإنسان إلى طرح التقليد الأعمى، وترير عقله منها، وبالتالي كان دور الكندي في التوفيق بين العقل والنقل مبنيا على أسس عقلية فلسفية وإسلامية.

\section{قائمة المراجع}

القرآن الكريم برواية قالون عن نافع المدني. أبوريان، محمد علي (1992). تاريخ الفكر الفلسفي. كلية الآداب، جامعة الإسكندرية، دار المعرفة الجامعية. 
أبوريان، محمد علي. (1990) ـ تاريخ الفكر الفلسفي من طاليس إلى أفلاطون. الإسكندرية: دار المعرفة الجامعية.

أحمد، هناء عبده سليمان. (2005). أثر المعتزلة في الفلسنة الإلمية عند الكندي. مكتبة

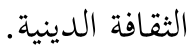

آل ياسين، جعفر. (1983). فيلسوفان رائدان (الكنادي والفارابي). بيروت: دار الأندلس للطباعة والنشر والتوزيع.

الإمام، زكريا بشير (1989). تاريخ الفلسفة الإسلامية. دار النشر السودانية للكتب. الأهواني، أحمد فؤاد (د.ت). الكندي فيلسوف العرب. سلسلة أعلام العرب، رقم 26،

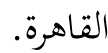

دي بور، ت.ج. (1981). تاريخ الفلسفة في الإسلام (ترجمة: محمد عبد الهادي أبو ريدة). بيروت: دار النهضة العربية للطباعة والنشر.

الشرقاوي، محمد عبد الله. (1980). مدخل نقدي إلى الفلسنة. بيروت: دار الجيل. الشيباني، عمر محمد التومي. (1975). مقدمة في الفلسغة الإسلامية. القاهرة: الدار

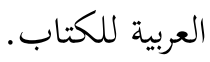

العراقي، محمد عاطف. (1972). دراسات في مذاهب الفلاسفة. القاهرة. علي، سيد أمير. (1938) غتتصر تاريخ العرب والتجديد الإسلامي. (ترجمة: رياض

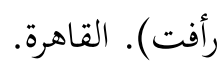

علي، عصام الدين محمد. (1994). تاريخ الفلسنة الإسلامية. الإسكندرية: دار

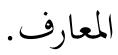

فرحان، محمد جلوب. (1986). دراسات في الفكر الفلسفي الإسلامي، كلية التربيةجامعة الموصل. 


$$
\text { فروخ، عمر . (1981). تاريخ الفكر العربي.ط3. بيروت دار العلم للملايين. }
$$

الكندي (1971)، رسائل الكندي الفلسغية، (تحقيق وضبط: الدكتور: محمد عبد الهادي أبوريدة). القاهرة.

كرم، يوسف. (1946). تاريخ الفلسغة اليونانية، القاهرة.

محمد، سماح رافع. (1971). تاريخ الفكر الفلسني في العصور القديمة والحلديثة. طرابلس: مؤسسة الفرجاني.

محمود، زكي نجيب. (1963). الموسوعة الفلسغية المختصرة. القاهرة: مكتبة الأنجلو

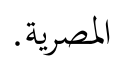

المهليل، السيد محمد عقيل بن عثمان. (1988). مدخل إلى الفلسفة. القاهرة: دار الحديث. 